\title{
NF-kappa B p65 phosphorylated at serine-536 is an independent prognostic factor in Swedish colorectal cancer patients
}

\author{
Andreas Lewander, Jingfang Gao, John Carstensen, Gunnar Arbman, \\ Hong Zhang and Xiao-Feng Sun
}

\section{Linköping University Post Print}

N.B.: When citing this work, cite the original article.

The original publication is available at www.springerlink.com:

Andreas Lewander, Jingfang Gao, John Carstensen, Gunnar Arbman, Hong Zhang and XiaoFeng Sun, NF-kappa B p65 phosphorylated at serine-536 is an independent prognostic factor in Swedish colorectal cancer patients, 2012, International Journal of Colorectal Disease, (27), 4, 447-452.

http://dx.doi.org/10.1007/s00384-011-1356-8

Copyright: Springer Verlag (Germany)

http://www.springerlink.com/?MUD=MP

Postprint available at: Linköping University Electronic Press http://urn.kb.se/resolve?urn=urn:nbn:se:liu:diva-76610 


\title{
NF-kB p65 Phosphorylated at Serine-536 Is an Independent Prognostic Factor in Swedish Colorectal Cancer Patients ${ }^{1}$
}

\author{
Andreas Lewander, Jinfang Gao, John Carstensen, Gunnar Arbman, \\ Hong Zhang, Xiao-Feng Sun ${ }^{2}$
}

Division of Oncology, Department of Clinical and Experimental Medicine (A.L., J.G., X-F.S.), Department of Health and Society (J.C.), Faculty of Health Sciences, Linköping University, Linköping, Sweden, Department of Surgery (G.A.), Country Council of Östergötland, Sweden, Tumour Biology (H. Z), School of Life Sciences, University of Skövde, Skövde, Sweden

Running Title: NF-kB p65 in Colorectal Cancer Patients

${ }^{1}$ The study was supported by grants from the Swedish Cancer Foundation, Swedish Research Council and the Health Research Council in the SouthEast of Sweden. 
${ }^{2}$ To whom requests for reprints should be addressed, at Division of Oncology, Department of Clinical and Experimental Medicine, Faculty of Health Sciences, Linköping University, SE-581 85 Linköping, Sweden.

Tel: +46-10-1032066; Fax: +46-10-1033090; E-mail address: xiaofeng.sun@liu.se 


\section{Abstract}

NF-kB transcription factor protein family has diverse cellular and biological functions, and post-translational modification is important to regulate these functions. An important site of phosphorylation of NF-kB p65 subunit is at Serine-536 (phospho-Ser536-p65), and this phosphorylation is involved in regulation of transcriptional activity, nuclear localization and protein stability. In this study, we investigated expression of phospho-Ser536-p65 in colorectal cancers and its relationships with clinicopathological factors. The expression of phospho-Ser536-p65 was examined by immunohistochemistry in 203 primary colorectal cancers, 156 normal mucosa specimens and 18 metastases in the lymph nodes. The expression of phospho-Ser536-p65 increased from normal mucosa to primary tumor $(p<0.0001)$. Further, the increased expression of phospho-Ser536-p65 in the cytoplasm of the primary tumors correlated with worse survival of the patients independently of gender, age, tumor location, stage and differentiation $(p=0.04$, hazard ratio $1.89,95 \% \mathrm{Cl}$ 1.03-3.47). The NF-kB p65 subunit phosphorylated at Serine-536 is an independent prognostic factor in colorectal cancer patients.

Key words: NF-кB, Survival, Colorectal cancer, Immunohistochemistry. 


\section{Introduction}

Colorectal cancer is one of the most common forms of cancers after prostate and breast cancer in the Western world. Despite extraordinary progress in survival due to improved diagnosis and especially treatment, many patients still suffer from recurrence and eventually die from the cancer. There is significant variation in the response to the treatments and survival among patients even though they have tumour in the same stage [1]. Therefore, it is very important to search for more biological markers to identify patient survival and the response to treatments.

NF-KB transcription factor protein family is involved in numerous cellular activities that, if not kept in balance, will transform the cell into a cancer cell $[2,3]$. Five members of the family have been found in human cells, RelA (p65), p105/p50, p100/p52, RelB and c-Rel. The most common form in human cells is $\mathrm{p} 65 / \mathrm{p} 50$ heterodimer $[2,4,5]$. The regulation of the NF-KB protein family is very important. Dimers of NF-kB are kept in the cytoplasm in the cells in an inactive state by binding to inhibitory proteins. Upon activation, inhibitory proteins are degraded and the proteins are translocated into the nucleus where they exert their effect. The regulation also occurs at the posttranslational level, where protein phosphorylation of the different subunits is a very important mechanism of regulation. Several different phosphorylation sites on the subunits have been discovered. An important site of phosphorylation of p65 subunit is at Serine-536 (phospho-Ser536-p65), and this phosphorylation is involved in regulation of transcriptional activity, nuclear localization and protein stability [6-9]. Studies have shown that the phosphoSer536-p65 can be found in both the cytoplasm and the nucleus depending on 
the stimulus, and the phosphorylation is probably also cell-type specific $[4,10$ 12].

In the light of recent knowledge of the importance of regulation of NF$\mathrm{KB}$ activity through phosphorylation we wanted to investigate whether expression of phospho-Ser536-p65 was related to colorectal cancer development, especially to clinicopathological variables, including survival, in patients. 


\section{Materials and methods}

\section{Patients}

Formalin-fixed paraffin-embedded tissue blocks were obtained from 203 patients with primary colorectal adenocarcinoma as the patients underwent surgical resection at Linköping Hospital or Vrinnevi hospital between 1972 and 2004. The study also included 156 normal mucosa specimens (of them 128 were matched with the primary tumors) taken from either the margin of distant resection or adjacent to the primary tumors, and 18 metastases in the lymph nodes (12 of them were matched with the primary tumors). The patients' gender, age, tumor location, stage and differentiation (better differentiation including well and moderate differentiation and poorer differentiation including poor differentiation, mucinous and signet-ring cell carcinomas) were obtained from surgical and/or pathological records at Linköping and Vrinnevi Hospitals. All patients were followed-up, and the mean period of the follow-up was 77 months (median, 56 months, and range, 0.06-288 months), the data were received/confirmed from/by surgeons and oncologist as well as the staff at Swedish Cancer Register Center, and the follow-up information was updated at least annually. The survival analyses were based on overall survival.

\section{Immunohistochemistry}

Five-micrometer sections were deparaffinised in xylene and rehydrated in graded ethanol. The method for antigen retrieval we used was high-pressure cooking in 0.01 M Tris-EDTA buffer ( $\mathrm{pH} 9.0$ ). The sections were heated to $125^{\circ} \mathrm{C}$ for $30 \mathrm{sec}$ and then cooled to $90^{\circ} \mathrm{C}$ for $10 \mathrm{sec}$, the sections were then kept in the buffer until room temperature. The sections were incubated with 
$3 \% \mathrm{H}_{2} \mathrm{O}_{2}$-methanol for 20 min and washed with phosphate-buffered saline (PBS, pH 7.4). After that the sections were incubated with rabbit anti-phosphoSer536-p65 antibody (Abcam, MA, the antibody binds specifically to the Ser536-phosphorylated form of p65 and does not cross-react with nonphosphorylated p65 or any other members of the NF-kB family) at $20 \mathrm{mg} / \mathrm{ml}$ in antibody diluent (Dako, Carpinteria, CA) overnight, followed by rinsing with PBS. Subsequently, the sections were incubated with a goat anti-rabbit secondary antibody coupled with peroxidise provided by the Dako ChemMate EnVision Detection Kit (Dako) for 25 min, and washed with PBS. The peroxidise reaction, using 3,3'-diaminobenzidine tetrahydrochloride (Dako), was performed for $8 \mathrm{~min}$. Sections known to stain positively were included as positive controls. The negative controls used PBS instead of the primary antibody. In all staining procedures, the positive controls showed clear staining, and there was no staining in the negative controls.

The sections were microscopically examined and scored independently by Lewander A and Gao J without any information on the clinicopathological data. The slides were initially classified, in a subjective matter, as negative ( $<5 \%$ of positive cells), weak, moderate and strong staining in the cytoplasm of normal epithelial cells, and tumor cells located in the entire area or invasive margin of primary tumor and metastasis, irrespective of the percentage of positive cells. For statistical analysis, we combined the negative cases with weak-stained cases as a group called weak expression, and combined moderate- and strong-stained cases as a group called strong expression, based on the similarities of the clinicopathological features. The expression in the nucleus of normal epithelial and tumor cells 
was classified as negative ( $<5 \%$ of the nucleus stained) or positive expression. To avoid artificial effects, cells in areas with necrosis, with poor morphology, or in the margins of sections were excluded from the analysis.

\section{Statistical analysis}

The significance of the difference in phospho-Ser536-p65 expression between normal mucosa, primary tumor and metastasis was tested by using McNemar methods. The relationships between phospho-Ser536-p65 expression and clinicopathological variables were tested by Pearson Chi-square method, and the relationships to survival were examined by using Wald Cox's proportional hazard model. Survival curves were calculated by using the Kaplan-Meier method. In the survival analyses, only patients who died from colorectal cancer were classified as events, while those who died from other causes or were still alive were considered as censored. Two-sided $p$ values of $<0.05$ were considered statistically significant. 


\section{Results}

Phospho-Ser536-p65 expression in the cytoplasm of normal mucosa, primary tumor and metastasis in the lymph node

Table 1 presents the frequency of phospho-Ser536-p65 cytoplasmic expression in normal mucosa, entire primary tumor, invasive margin of primary tumor (excluding 27 cases that did not have visible invasive margin), and metastasis in the lymph node. We compared the staining intensity of phosphoSer536-p65 in normal mucosa with entire primary tumor in the 128 matched cases by using the McNemar test. There were significantly more samples having strong expression among the primary tumors compared to normal mucosa $(p<0.0001)$, i.e., among the 128 cases, 56 cases had weak expression in normal tissue but strong expression in tumor, in contrast, only 5 cases had strong expression in normal tissue, but weak expression in tumor, the rest (67 cases) had identical expression in both normal and tumor tissue. There was no significant difference in the frequency of the expression between entire primary tumors and metastasis in the 12 matched cases $(p=0.22)$.

We also compared the staining intensity of phospho-Ser536-p65 in normal mucosa with that in invasive margin of primary tumor by using the McNemar test, the results were similar to a comparison of the normal mucosa with entire primary tumor, i.e., the frequency of the strong staining was higher in the invasive margin of primary tumor than in normal mucosa $(p<0.0001)$.

Figure 1 shows phospho-Ser536-p65 expression in normal mucosa, primary tumor and metastases in the lymph node. There was no phosphoSer536-p65 expression in normal mucosa but strong expression in the cytoplasm of primary and metastatic tumors. 
Table 1 Phospho-Ser536-p65 expression in the cytoplasm of normal mucosa, primary tumour, and metastasis in the lymph node.

\section{Phospho-Ser536-p65 expression}

Weak (\%)

Strong (\%)

Normal mucosa $(n=156)$

$107(69)$

$49(31)$

Primary tumour

Entire tumour $(n=203)$

$73(36)$

$130(64)$

Invasive margin $\left(n=176^{*}\right)$

$60(34)$

$116(66)$

Metastases $(n=18)$

$10(56)$

$8(44)$

* Excluding 27 cases that did not have a visible invasive margin.

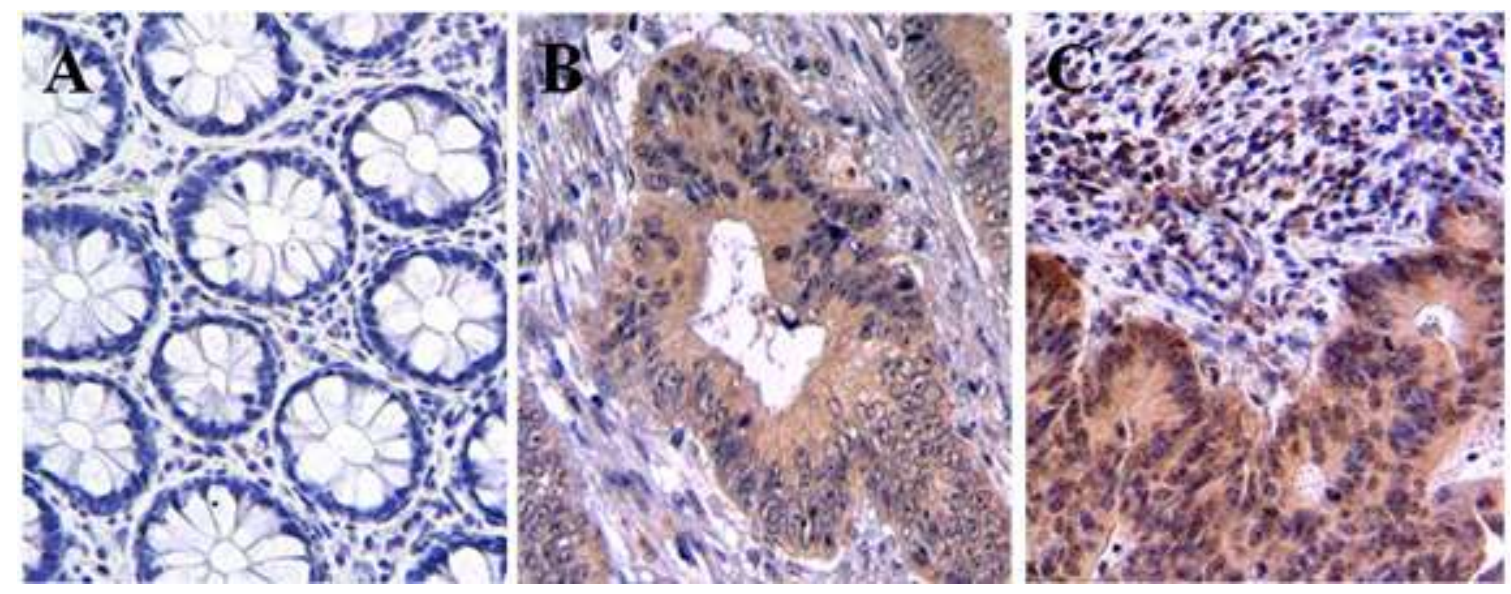

Fig. 1 - Phospho-Ser536-p65 expression was negative in normal mucosa (A), strong in primary tumor $(B)$ and strong in metastases in the lymph node $(C)$. 


\section{Phospho-Ser536-p65 expression in the cytoplasm in relation to clinicopathological variables}

We first analyzed the relationship of cytoplasmic phospho-Ser536-p65 expression in the entire area of the primary tumor with survival. Univariate survival analysis showed that the strong expression of the phospho-Ser536p65 correlated with worse survival of the patients ( $p=0.02$, Figure 2$)$. In the further multivariate analysis, the prognostic significance still remained after adjusting for patients' gender, age, tumor location, stage and differentiation $(p=0.04$, hazard ratio $1.89,95 \% \mathrm{Cl} 1.03-3.47$, Table 2$)$. However, we did not find any statistically significant relationship of the phospho-Ser536-p65 expression with gender, age, tumor location, stage or differentiation by using Pearson Chi-square method ( $p>0.05$, data not shown).

Then we analyzed the relationship of cytoplasmic phospho-Ser536p65 expression in the invasive area of the primary tumor with survival. In the multivariate analysis, strong expression of the phospho-Ser536-p65 in the invasive margin of tumors was also related to worse survival independently of tumor stage $(p=0.04$, data not shown). However the prognostic significance was lost when we included patients' gender, age, tumor location and differentiation ( $p=0.13$, by Wald multivariate Cox regression analysis, data not shown). 

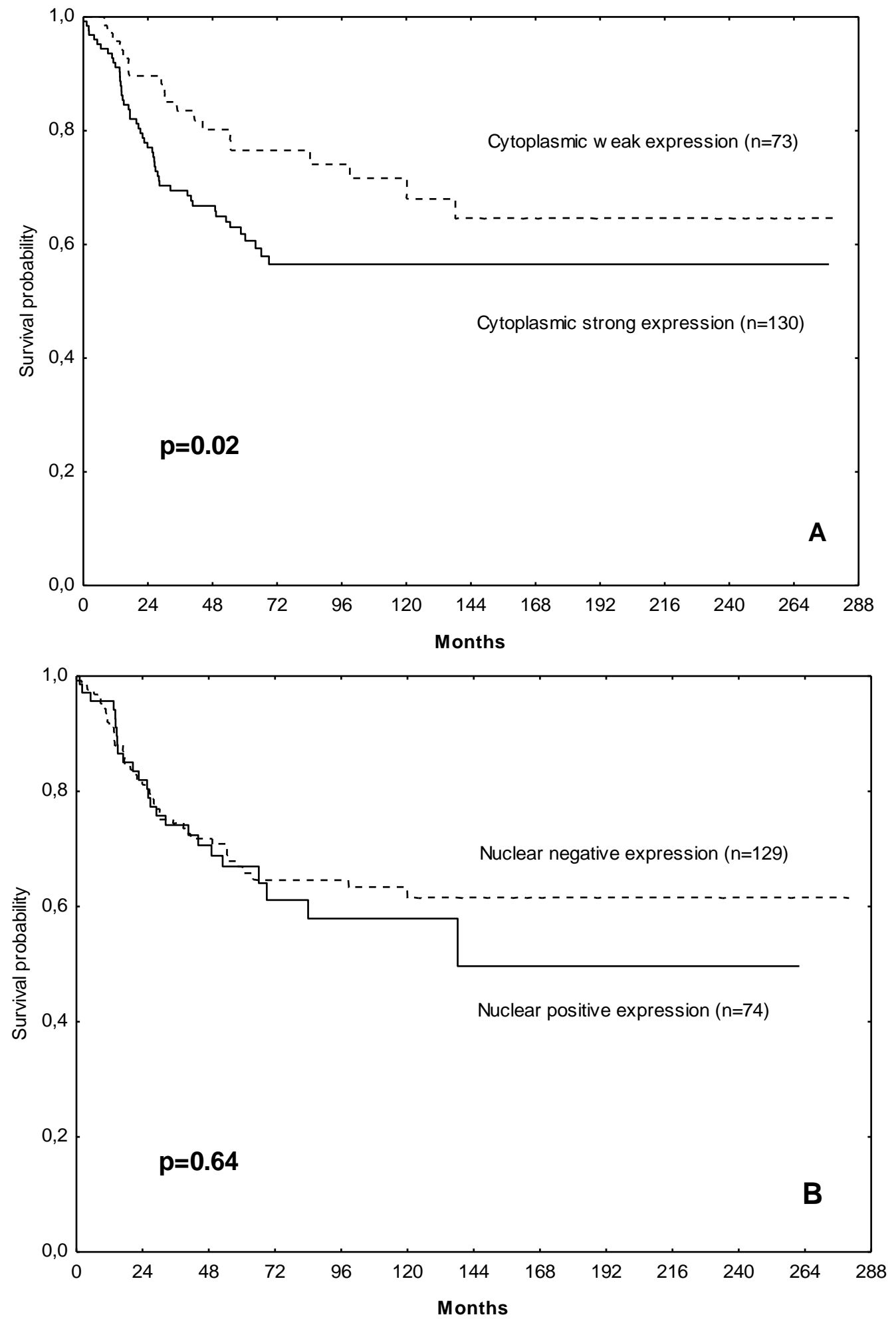

Fig. 2 - Phospho-Ser536-p65 expression in the cytoplasm (A) or in the nucleus (B) of the primary tumor in relation to survival in the patients with colorectal cancer. 
Table 2 Multivariate Cox regression analysis of phospho-Ser536-p65 expression in the cytoplasm, gender, age, tumour location, stage and differentiation to survival in colorectal cancer

\begin{tabular}{lllll}
\hline variable & No of patients & $\begin{array}{l}\text { Cancer death } \\
\text { Hazard ratio }\end{array}$ & $95 \% \mathrm{Cl}$ & $\mathrm{p}$-value
\end{tabular}

Phospho-Ser536-p65

0.04

Weak

72

1.00

Strong

123

1.89

$1.03-3.47$

Gender

Male

104

1.00

Female

91

1.18

0.70-1.97

Age (years)

74

1.00

$\geq 70$

121

1.52

$0.87-2.68$

Tumour location

Colon

98

1.00

Rectum

97

0.67

$0.39-1.14$

Stage

26

1.00

II

83

1.93

0.42-8.80

III

53

6.03

1.35-26.91

IV

33

38.3

8.77-167.91

Differentiation

Better

138

1.00

Poorer

57

1.18

0.14

0.14

0.54

0.14

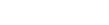




\section{Expression of phospho-Ser536-p65 in the nucleus}

There was a clear positive relationship between phospho-Ser536-p65

expression in the cytoplasm and that in the nucleus $(p<0.001$, by using Pearson Chi-square test, Table 3).

There was no statistical difference in the frequency of phosphoSer536-p65 expression in the nuclei from normal mucosa to primary tumors $(p=0.20$, by McNemar test), and from primary tumor to metastasis in the lymph node ( $p=0.29$, by McNemar test), neither with survival by using Wald Cox's proportional hazard model $(p=0.64$, Figure $2 \mathrm{~B})$ nor other clinicopathological variables by using Pearson Chi-square method ( $p>0.05$, data not shown). 


\section{Discussion}

NF-KB regulates the transcription of many genes which are responsible for immune response, cell adhesion, differentiation, proliferation, angiogenesis and apoptosis [2,11]. Abnormalities in NF-KB regulation are involved in multiple human pathologies including tumors. The function of NF-KB is inhibited by binding to nuclear factor-KB inhibitor (IKB) and the release of activated NF-KB follows proteasome-mediated degradation of IKB resulting from phosphorylation of the inhibitor and finally conjugation with ubiquitin $[2,10,11]$. Since NF-KB is responsible for the regulation of many other genes, changed expression of the NF-KB and IKB proteins could be potentially involved in tumor development. In the present study, we found that expression of NF-kB p65 phosphorylated at Serine-536 was increased in primary tumor compared to normal mucosa. However, the frequency of the protein expression in the metastases in lymph nodes was not different from primary tumor. Unregulated NF-KB in primary tumor compared with normal tissue has been found in previous studies. Lind et al [13] used an electrophoretic mobility shift assay technique and demonstrated that NF-KB in primary tumor was greatly increased compared with adjacent normal tissue from the same patients. Yu et al [14] examined the expression of NF-KB p65 by using a monoclonal antibody against NF-KB p65 in normal colorectal mucosa, colorectal adenomas and colorectal adenocarcinomas, and showed that NFKB p65 expression was significantly increased from normal mucosa to adenoma and to adenocarcinoma, furthermore the expression was increased with the transition from low to moderate and to high dysplasia of adenoma. 
Taken together, these results indicate that the NF-KB p65 may play a role in earlier development of colorectal cancer.

The most interesting finding in the present study, also the first report, is that the expression of phospho-Ser536-p65 in the cytoplasm was significantly correlated to survival in the patients with colorectal cancer. The strong expression predicted worse survival of the patients independently of patients' gender, age, tumor location, stage and differentiation. Even though the $p$ value is marginal $(p=0.04)$ hazard ratio $1.89(95 \% \mathrm{Cl} 1.03-3.47)$ is nearly two-times higher when comparing the strong expression with the weakexpression group.

Unexpectedly, the NF-kB p65 in the nucleus did not give clinicopathological significance. Several other studies in colorectal and pancreatic cancers also showed cytoplasmic expression of NF-kB p65, and further experiments demonstrated that the majority of NF-kB p65 which is freed from IkB remained in the cytoplasm [14-16]. During activation, NF-kB p65 is phosphorylated at several residues. What residues, when and where seem to be dependent on different stimuli that activate NF-kB [4]. There was a clear positive relationship between phospho-Ser536-p65 expression in the cytoplasm and that in the nucleus in the present study $(p<0.001$, Table 3$)$, it may suggest that when the phosphorylated cytoplasmic NF-kB is accumulated to a certain level, it will transfer to the nucleus. We did not see a direct relationship between nuclear NF-kB and survival, which may depend on a matter of time point and/or the number of the cases etc. In fact, in the survival curves of the nuclear NF-kB we saw a trend of the positive NFkB expression resulting in worse survival (similar to the cytoplasmic expression in relation to 
Table 3 Relationship between cytoplasm and nuclear staining in primary tumour.

\begin{tabular}{|c|c|c|c|}
\hline \multirow{2}{*}{$\begin{array}{l}\text { Cytoplasmic } \\
\text { expression }\end{array}$} & \multicolumn{2}{|c|}{ Nuclear expression } & \multirow{2}{*}{$p$-value* } \\
\hline & Negative (\%) & Positive (\%) & \\
\hline Weak $(n=73)$ & $58(79)$ & $15(21)$ & $<0.0001$ \\
\hline Strong $(n=130)$ & $71(55)$ & $59(45)$ & \\
\hline
\end{tabular}

* by Pearson Chi-square test,

survival). Sakurai et al. [6] was first to report that upon tumor necrosis factoralfa induced activation, IKB kinase phosphorylates NF-KB p65 at Serine-536 and that this occurs in the cytoplasm of HeLa cells before activated p65 translocates into the nucleus. Our present result showing the cytoplasmic staining by the antibody against phospho-Ser536-p65 further confirmed that phosphorylated NF-KB p65 was in the cytoplasm of colorectal cancers, as well as in the nucleus. It has been shown that even under long-term stimulation, only up to $20 \%$ of NF-KB p65 moved to the nucleus and the rest of the NF-KB p65 still remained in the cytoplasm [16]. Possible explanations for this phenomenon are 1) IKB is mutated and lost its binding to NF-kB p65, and masks the nuclear translocation signal in NF-kB p65, and 2) NF-kB p65 is mutated and inhibited its binding to IKB. Either mutation in IKB or NF-kB p65 prohibits their interaction, resulting in the changed activation and 
mislocalisation of NF-KB p65. We did not exclude a possibility that other biological factors which are related to cytoplasmic NF-kB p65 play a role (or more important role) in colorectal cancer development/survival, rather than the cytoplasmic NF-kB p65 itself, i.e., the cytoplasmic NF-kB p65 may be a secondary event (as a bystander effect) due to co-activation of other biological factors.

In summary, we found increased levels of NFKB p65 phosphorylated at Serine-536 in the cytoplasm of primary tumours compared with normal mucosa in colorectal cancer patients, however, we did not see a further increase from primary tumours to metastases in the lymph nodes, indicating that up-regulation of this protein is an early event in the development of colorectal cancer. Further, the cytoplasmic expression of NF-kB p65 phosphorylated at Serine-536 predicts a worse prognosis independent of patients' gender, age, tumour location, stage and differentiation. 
Conflicts of interest

None declared. 


\section{Acknowledgements}

The authors thank Helen Richard, Cecilia Bergenwald, Gertrud Stridh, Gunnel Lindell and Kerstin Ingels, Department of Pathology, Linköping Hospital, Sweden, for kindly preparing tissue sections, and Dr. David Hinselwood (a Scottish doctor) at the Division of Oncology, Linköping University, Sweden, for revising this paper. 


\section{References}

[1] Chee, C.E., and Sinicrope, F.A. Targeted therapeutic agents for colorectal cancer. Gastroendoterol. Clin. North Am., 39: 601-613, 2010.

[2] Sun X-F., and Zhang H. NFKB and NFKBI polymorphisms in relation to susceptibility of tumour and other diseases. Histol. Histopathol., 22: 13871398, 2007.

[3] Hanahan, D., and Weinberg, R.A. The hallmarks of cancer. Cell, 100: 57-70, 2000.

[4] Viatour, P., Merville, M.P., Bours. V., and Chariot., A. Phosphorylation of NFkappaB and lkappaB proteins: implications in cancer and inflammation. Trends Biochem. Sci., 30: 43-52, 2005.

[5] Moynagh, PN. The NF-kappaB pathway, J. Cell Sci., 118: 4589-4592, 2005.

[6] Sakurai, H., Chiba, H., Miyoshi, H., Sugita, T., and Toriumi, W. IkappaB kinases phosphorylate NF-kappaB p65 subunit on serine 536 in the transactivation domain. J. Biol. Chem., 274: 30353-30356, 1999.

[7] Sizemore, N., Lerner, N., Dombrowski, N., Sakurai, H., and Stark, G.R. Distinct roles of the IKB kinase $\alpha$ and $\beta$ subunits in liberating nuclear factor $\mathrm{KB}$ (NF-KB) from IKB and in phorphorylating the p65 subunit of NF-KB. J. Biol. Chem., 277: 3863-3869, 2002.

[8] Buss, H., Dörrie, A., Schmitz, M.L., Hoffmann, E., Resch, K., and Kracht, M. Constitutive and interleukin-1-inducible phosphorylation of p65 NF-kB at serine 536 is mediated by multiple protein kinases including IKB kinase (IKK)- $\alpha$, IKK $\beta$, IKKE, TRAF family member-associated (TANK)-binding kinase 1 (TBK1), and an unknown kinase and couples p65 to TATA-binding protein-associated factor II31-mediated interleukin-8 transcription. J. Biol. Chem. 279(2004) 55633-55643. 
[9] Lawrence, T., Bebien, M., Liu, G.Y., Nizet, V., and Karin, M. IKKa limits macrophage NF-KB activation and contributes to the resolution of inflammation. Nature, 434:1138-1143, 2005.

[10] Berenson, J.R., Ma, H.M., and Vescio, R. The role of nuclear factor-kappaB in the biology and treatment of multiple myeloma. Semin. Oncol., 28: 626-633, 2001.

[11] Beinke, S., and Ley, S.C. Functions of NF-kappaB1 and NF-kappaB2 in immune cell biology, Biochem. J., 382: 393-409, 2004.

[12] Yu, Y., Wan, Y., and Huang, C. The biological functions of NF-kappaB1 (p50) and its potential as an anti-cancer target. Curr. Cancer Drug Targets, 9:566$571,2009$.

[13] Lind, D.S., Hochwald, S.N., Malaty, J., Rekkas, S., Hebig, P., Mishra, G., Moldawer L.L., Copeland, EM $3^{\text {rd }}$., and S. Mackay. Nuclear factor-KB is upregulated in colorectal cancer. Surgery, 130: 363-369, 2001.

[14] Yu H.G ., Yu, L.L., Yang, Y., Luo, H.S., Yu, J.P., Meier, J.J., Schrader, H., Bastian, A., Schmidt, W.E., and Schmitz, F. Increased expression of RelA/nuclear factor-kappa B protein correlates with colorectal tumorigenesis. Oncology, 65:37-45, 2003.

[15] Wang, W., Abbruzzese, J.L., Evans, D.B., Larry, L., Cleary, K.R., and Chiao, PJ. The nuclear factor-kappa B RelA transcription factor is constitutively activated in human pancreatic adenocarcinoma cells. Clin. Cancer Res., 5: 119-127, 1999.

[16] Verma, I.M., Stevenson, J.K., Schwarz, E.M., Van Antwerp, D., and Miyamoto, S. Rel/NF-kappa B/I kappa B family: intimate tales of association and dissociation. Genes Dev., 9: 2723-2735, 1995. 
[17] Miyamoto, S., Chiao, P.J., and Verma, IM. Enhanced I kappa B alpha degradation is responsible for constitutive NF-kappa B activity in mature murine B-cell lines. Mol. Cell Biol., 14: 3276-3282, 1994. 